\section{Inquérito sociocomportamental sobre as práticas sexuais desprotegidas entre homens que fazem sexo com homens usuários da Internet}

\section{Internet behavior survey about the unprotected anal sex with men who have sex with men}

\author{
Sandra Brignol' \\ Inês Dourado' \\ ' Instituto de Saúde Coletiva da Universidade Federal da Bahia (UFBA) - \\ Salvador (BA), Brasil
}

\section{Resumo}

Introdução: $\mathrm{O}$ sexo anal sem o uso do preservativo masculino é um importante fator de risco para a infecção por HIV e outras doenças sexualmente transmissíveis. Objetivo: Investigar fatores individuais e sociais que se relacionam ao sexo anal desprotegido entre homens que se autodeclararam HIV negativos e que fizeram sexo anal com parceiros HIV positivos ou de sorologia desconhecida. Metodologia: Este estudo é parte de um inquérito sociocomportamental entre homens que fazem sexo com homens (HSH), a coleta de dados foi on-line no site do projeto Convida. A amostra deste estudo foi de $487 \mathrm{HSH}$. A análise descritiva precedeu a análise bivariada com o uso do teste $\chi^{2}$. Para a modelagem utilizou-se a regressão logística. Resultados: Praticaram sexo anal desprotegido com parceiro HIV positivo ou de sorologia desconhecida no último ano $30,8 \%$ dos participantes, sendo a maioria de classes sociais mais elevadas, brancos e solteiros. Os fatores que se associaram à prática do sexo anal sem proteção foram: ter mais de três parceiros no último ano (OR=1,7; IC95\% 1,1-2,7), autopercepção de alto ou médio risco para adquirir HIV/Aids (OR=2,7; IC95\% 1,5-3,6) e gostar de sexo oral passivo ( $\mathrm{OR}=0,3$; IC95\% 0,2 $0,8)$. Conclusão: O sexo anal desprotegido é uma prática frequente entre os HSH entrevistados. A frequência a banheiros públicos deve ser explorada enquanto fator de risco para o envolvimento no sexo anal sem proteção. Outros estudos on-line são necessários e importantes para o entendimento dessas relações e do envolvimento em práticas sexuais arriscadas para infecção por HIV entre HSH.

Palavras-chave: HIV; vulnerabilidade; comportamento sexual; homossexualidade masculina; internet; assunção de riscos.

Trabalho realizado na Universidade Federal da Bahia (UFBA) - Salvador (BA), Brasil. 
Abstract

Introduction: Unprotected anal sex (insertive or receptive) is an important risk factor for HIV infection and other STDs. Objetive: The objective was to investigate individual and social factors associated with unprotected anal sex among Men Who have Sex with Men (MSM) that declared being HIV negative and engaged in anal sex with an HIV positive partner or of ignored serology. Methods: This study is part of a survey among MSM and among Internet users, with data collected from an on line questionnaire in project site. The internet sample for this study was 487 MSM. Descriptive and stratified analyses were carried out and $\chi^{2}$ test was used. Logistic regression was used for modeling the data. Results: Among the participants $30.8 \%$ referred unprotected anal sex in the previous 12 months with an HIV positive partner or partner with unknown serology. Most of them belonged to higher social classes, were white and single. Factors associated with unprotected anal sex were: number of partners (more than 3 ) in the previous 12 months ( $\mathrm{OR}=1.7$; 95\% CI 1.1-2.7), high or medium perception of risk to HIV/Aids ( $\mathrm{OR}=2.7$; 95\% CI 1.5-3.6) and enjoy passive oral sex $(\mathrm{OR}=0.3 ; 95 \%$ CI 0.2-0.8). Conclusion: Unprotected anal sex was a frequent practice among MSM in this survey. The frequency to public bathrooms should be further explored as a risk factor for unprotected anal sex. It is important to further explore these findings in order to understand these relationships and the involvement in risky sexual practices for HIV infection among MSM.

Keywords: HIV; vulnerability; sexual behavior; homosexuality; internet ; risk-taking .

\section{Introdução}

O sexo anal (ativo ou passivo) sem o uso do preservativo masculino é um importante fator de risco para a infecção por HIV e outras doenças sexualmente transmissíveis (DSTs). Na literatura, alguns estudos que investigaram as práticas e comportamentos sexuais de homens que fazem sexo com homens (HSH), os quais recrutam parceiros sexuais na internet, mostram resultados que indicam que a internet proporciona certa facilidade para o encontro de parceiros sexuais e para o sexo anal desprotegi$\mathrm{do}^{1-6}$. Por outro lado, outro estudo ${ }^{7}$, comparando $\mathrm{HSH}$ que buscaram parcerias na internet com HSH que buscaram parcerias fora da rede, mostrou maior prevalência do sexo arriscado para HIV no último grupo.

No Brasil, são raros os estudos sociocomportamentais via internet, e, além disso, explora-se pouco as potencialidades desse "ambiente" para investigações sobre as práticas sexuais de risco para infecção por HIV $^{8}$. Recentemente, uma pesquisa qualitativa utilizou o potencial da internet para investigar práticas de sexo anal desprotegido de forma deliberada (barebaking) entre $\mathrm{HSH}^{9}$. E em outros países, as pesquisas na internet começaram ser utilizadas a partir do ano 2000, e desde então tem crescido o número de investigações quantitativas que recrutam participantes homens homo e bissexuais na internet ${ }^{5}$.

Em relação aos usuários da internet no Brasil, em 2004, ano em que foi iniciada a pesquisa, $13,7 \%$ dos domicílios brasileiros tinham acesso à internet, entre as pessoas que acessavam ou usavam a rede, $22 \%$ eram homens e $20,2 \%$ eram mulheres e que quanto mais elevado o nível de instrução e rendimento mensal domiciliar per capita, maior era a proporção de pessoas que acessavam a internet ${ }^{10}$. Na faixa de idade acima de 25 anos, os homens acessam mais a internet quando comparados às mulheres ${ }^{11}$. Em 2005, 16,0\% dos domicílios tinham acesso à internet e $28,0 \%$ dos que acessavam a rede eram homens e $22 \%$ eram mulheres ${ }^{12}$. Hoje, $23,8 \%$ dos domicílios no Brasil têm acesso à 
internet ${ }^{13}$ e o perfil de usuário tem se mantido nas faixas de renda acima de três salários mínimos, com ensino médio e superior completo, e local de acesso padrão o domicílio seguido do local de trabalho e instituições de ensino.

No contexto da epidemia do HIV e Aids, para ações de prevenção é importante descrever e identificar determinantes da prática do sexo anal sem o uso do preservativo. O aumento das práticas sexuais de risco para infecção por HIV, verificado em alguns estudos ${ }^{1,5}$ por vezes, não apresentam o conhecimento da sorologia dos parceiros. Além disso, na cidade de Salvador, são raros os estudos que apresentam este enfoque. Assim, objetivou-se estudar, em uma população de HSH potencialmente soronegativa para o HIV, a associação entre os fatores individuais e sociais e a prática do sexo anal desprotegido com parceiros sabidamente HIV positivos ou de sorologia desconhecida, visto que o conhecimento da sorologia negativa do parceiro, para HIV, pode ser um dos determinantes da não adoção de práticas mais seguras.

\section{Métodos}

Realizou-se um inquérito sociocomportamental com o objetivo de produzir conhecimento sobre aspectos da sexualidade, modo de vida, práticas de risco e de prevenção para a infecção por HIV, na internet entre HSH usuários de sites gays, no país - Projeto Convida. A coleta de dados foi realizada no site específico do Projeto (www.convida.org.br), entre junho de 2003 e janeiro de 2006, período em que o site e um questionário on-line, anônimo padronizado autorrespondido, com 68 perguntas, esteve disponível na web. A divulgação do projeto, convidando os homens para responder ao questionário, foi feita em revistas gays e sites de instituições do movimento social gay. Cartazes e panfletos sobre o projeto e com endereço do site foram distribuídos em locais de sociabilidade gay, bem como nas Paradas do Orgulho Gay de Salvador e São Paulo em
2003. Este site, além de viabilizar a coleta de informações, mobilizou outras estratégias de promoção da saúde, contextualizando as novas modalidades de hábitos e práticas sexuais, abrangendo outras informações (educativas e culturais) de interesse do público gay. Também foi disponibilizado para o movimento social banner animado para divulgação da pesquisa. $\mathrm{O}$ questionário quando concluído era automaticamente enviado para uma base de dados no programa Microsoft Access. Outra parte da coleta de dados do projeto foi realizada em Salvador, nos locais frequentados por essa população (bares, boates, videolocadoras, barraca de praia, saunas, etc.), porém, estes últimos dados não foram analisados neste trabalho. Como se tratava de um estudo exploratório, inédito e de mapeamento, baseado em um estudo realizado na França, não foi calculado um tamanho mínimo de amostra para o estudo original ${ }^{13}$.

Para essa análise foram selecionados homens que praticaram sexo anal desprotegido pelo menos uma vez nos últimos 12 meses, declararam ser HIV negativos e se autodefiniram como homo e bissexuais. A Figura 1 apresenta a composição da amostra segundo os critérios de inclusão do estudo.

A variável de desfecho, denominada "sexo anal desprotegido no último ano", ou seja, sem uso de preservativo, foi categorizada em sexo anal desprotegido com parceiros sabidamente HIV positivos ( $\mathrm{n}=12$ ) ou de sorologia desconhecida $(\mathrm{n}=129) e$ sexo anal desprotegido com parceiros de sorologia negativa $(n=369)$; não responderam à pergunta sobre a sorologia do parceiro 23 homens. Como estas variáveis foram baseadas em respostas de relato, pode haver um viés, pois não tínhamos como verificar se o relato da sorologia era a verdadeira sorologia do participante e seus parceiros. Utilizou-se o quadro conceitual de vulnerabilidade ${ }^{14}$ adaptado para o contexto da epidemia no Brasil ${ }^{15}$, para nortear a seleção das covariáveis do estudo em dois grandes grupos de fatores, os sociais e os individuais, que são apresentados nas tabelas de 


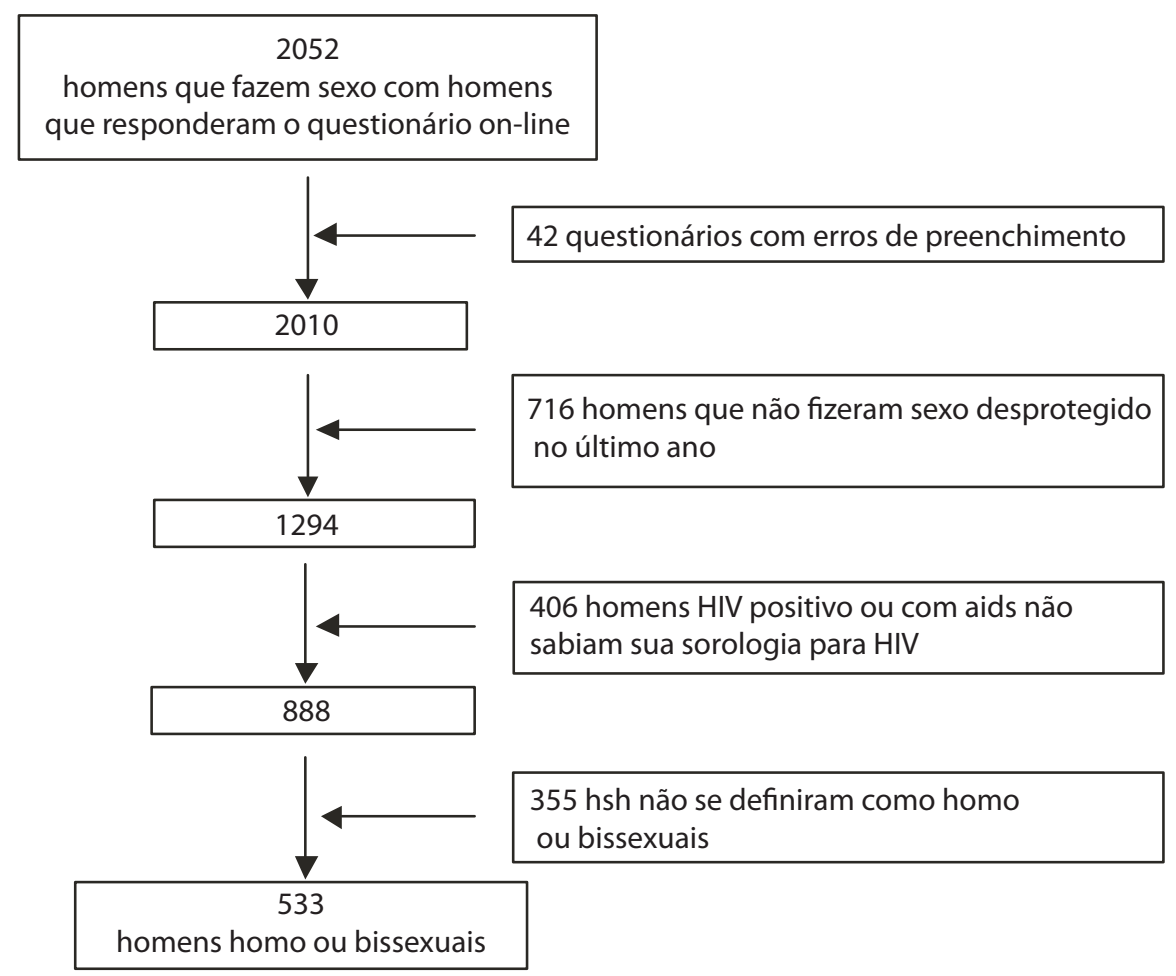

Figura 1. Composição da amostra do estudo

Figure 1. Composition of the sample

resultados. A escolha dos locais de sociabilidade gay (bares, boates, videolocadoras, barracas de praia, saunas etc.), baseou-se na literatura que aponta que frequentar alguns desses locais pode estar associado ao envolvimento em práticas de risco para infecção por $\mathrm{HIV}^{2,27}$. As classes sociais alta, média $\mathrm{e}$ baixa foram construídas segundo os quartis dos coeficientes do Indicador Econômico Nacional (IEN) ${ }^{16}$.

$\mathrm{O}$ ponto de corte para categorizar o número de parceiros não é padronizado na literatura, onde encontramos valores acima de 20, ou acima de 10, ou acima de cinco ou ainda acima de um parceiro, para definir a categoria de maior risco para o envolvimento na prática do sexo anal desprotegido. Neste estudo usamos o ponto de corte de três parceiros sexuais nos últimos 12 meses, baseando-se no primeiro quartil da distribuição da variável, pois a distribuição era muito assimétrica. Para as demais variáveis contínuas usamos a média aritmética para as comparações.
Na análise descritiva das variáveis categorizadas, usaram-se os testes $\chi^{2} \mathrm{e}$ exato de Fisher, para as variáveis contínuas os testes $t$ de Student e Man-Whitney. O nível de significância estatística para todos os testes foi de 0,05 . A análise multivariada foi realizada com regressão logística para avaliar a associação entre os fatores de vulnerabilidade e o sexo anal desprotegido. Para as não respostas foram criadas as categorias "sem resposta", para cada variável analisada. O Projeto Convida foi submetido e aprovado pelo Comitê de Ética e Pesquisa do Instituto de Saúde Coletiva da Universidade Federal da Bahia, não havendo conflito de interesse vinculado a esta pesquisa.

\section{Resultados}

\section{População do estudo}

Entre os 533 participantes que atenderam aos critérios de inclusão no estudo, a idade média dos participantes foi de 30,5 
$( \pm 9,5)$ anos. O número médio de parceiros homens no último ano foi de 8,6 ( \pm 35 ) parceiros, sendo que $50 \%$ dos participantes tiveram até 12 parceiros homens no último ano.

Em relação às características socioeconômicas, verificou-se que a maioria $(87,4 \%)$ foi classificada como sendo da classe alta; autodefiniram-se de raça branca $(71,5 \%)$, com graduação incompleta (31,5\%); graduação completa $(27,2 \%)$ e com pós-graduação $19,5 \%$. Solteiros, divorciados ou separados foram $68,7 \%$ (Tabela 1 ).

A média de idade da primeira experiência homossexual "sem penetração" foi 15,7 $( \pm 5,6)$ anos e da primeira experiência homossexual "com penetração" a média de idade foi 16,3 $( \pm 5,3)$ anos. Comparando-se a média de idade da primeira experiência "sem penetração" entre o grupo de homens que praticaram sexo anal com parceiro HIV positivo ou de sorologia desconhecida nos últimos 12 meses, essa média foi de $14,8( \pm 5,3)$ anos e para os homens que praticaram sexo anal com parceiros de HIV negativos foi de 16,2 $( \pm 5,6)$ respectivamente, sendo a diferença estatisticamente significante $(\mathrm{p}=0,024)$.

A pratica do sexo anal (ativo ou passivo) desprotegido com parceiro HIV positivo ou de sorologia desconhecida foi relatada por $30,8 \%$ dos participantes; $68 \%$ declararam ter tido relações sexuais com homens que conheceram na internet; $88,2 \%$ referiram ter feito o teste para o HIV pelo menos uma vez na vida; $26,1 \%$ disseram sentir prazer em situações que envolvem algum tipo de perigo durante as relações sexuais; e 34,7\% disseram gostar de fazer sexo em público; $18,2 \%$ participaram de atividades em instituição social e/ou organização não governamental. Quanto aos locais de sociabilidade gay, $12,4 \%$ dos indivíduos disseram frequentar sempre ou frequentemente saunas, 9,4\% quartos-escuros e $14,4 \%$ videolocadoras (Tabela 1).

Na análise bivariada, os fatores individuais que se associaram significativamente com a prática do sexo anal sem proteção com parceiros HIV positivos ou com sorologia desconhecida foram: número de parceiros no Carnaval $(\mathrm{p}=0,026)$; número de parceiros no ano ( $p=0,0001)$; sentir-se em risco de contrair HIV/
Aids ( $\mathrm{p}=0,0001)$; sexo oral passivo $(\mathrm{p}=0,038)$; ter feito o teste para HIV, ( $\mathrm{p}=0,018)$; frequentar saunas $(\mathrm{p}=0,007)$; banheiros públicos $(\mathrm{p}=0,001)$; cinema pornô $(\mathrm{p}=0,019)$ (Tabela 2). Porém, na análise multivariada, permaneceram significativas as seguintes associações: número de parceiros no último ano (OR=1,71; IC 95\% 1,1-2,7); sentir-se em risco de contrair HIV ou AIDS (OR=2,7; IC 95\% 1,5-3,6) e gostar de sexo oral passivo (OR=0,5; IC 95\% 0,2-0,8). Os demais fatores não atingiram níveis de significância estatística (Tabela 3).

Ainda na fase da análise exploratória, alguns resultados, relacionados à prática do sexo anal sem o uso do preservativo, chamaram a atenção, por exemplo, verificou-se que dentre os homens que sabiam ser HIV positivos ou com AIDS ( $n=93$ ), 51,4\% relataram sexo anal desprotegido nos últimos 12 meses anteriores ao preenchimento do questionário. Na análise geral, com todos os participantes, o conhecimento da sua própria sorologia do HIV é suposta, pois somente $41 \%$ disseram ter realizado o exame várias vezes na vida, e $15 \%$ nunca fizeram o teste para HIV, além disso, não houve comprovação da sorologia dos parceiros, acreditando apenas no relato destes.

\section{Discussão}

A prevalência de $30,8 \%$ de relato de envolvimento na prática do sexo anal desprotegido, com homens HIV positivos ou de sorologia desconhecida, é relativamente alta. Esse nível de prevalência foi observado em quatro estudos ${ }^{6,21,23,32}$, porém, outros estudos descreveram prevalências bem menores como $12,7 \%$ (seis estudos) e $8 \%$ (sete estudos).

Entre as características que identificam os HSH que participaram da pesquisa e responderam ao questionário na internet, a maioria fez sexo com parceiros que conheceu na rede, tinham alta escolaridade, eram predominantemente brancos e solteiros morando com amigos ou familiares. Poucos declararam morar com companheiros. Características similares foram descritas por outros estudos nacionais $^{8,33,34}$ e internacionais ${ }^{2,3,5,7,23}$ entre 
Tabela 1. Características socioeconômicas, demográficas e culturais entre homens que fazem sexo com homens

Table 1. Socioeconomic, demographic, and cultural characteristics among men who have sex with men (MSM)

\begin{tabular}{|c|c|c|}
\hline Variável & $\mathrm{n}$ & $\%$ \\
\hline \multicolumn{3}{|l|}{ Sexo anal desprotegido no ano } \\
\hline Com parceiros de sabidamente HIV negativo & 369 & 69,2 \\
\hline Com parceiros HIV + ou sorologia desconhecida & 164 & 30,8 \\
\hline Total & 533 & 100,0 \\
\hline \multicolumn{3}{|l|}{ Você faz parte de alguma associação } \\
\hline Sim & 97 & 18,2 \\
\hline Não & 423 & 79,4 \\
\hline Sem resposta & 13 & 2,4 \\
\hline Total & 533 & 100,0 \\
\hline \multicolumn{3}{|l|}{ Transou com homens que conheceu pela Internet } \\
\hline Sim & 364 & 68,3 \\
\hline Não-Não usa Internet & 167 & 31,3 \\
\hline Sem resposta & 2 & 0,4 \\
\hline Total & 533 & 100,0 \\
\hline \multicolumn{3}{|l|}{ Você já fez o teste do HIV? } \\
\hline Sim & 470 & 88,2 \\
\hline Não & 62 & 11,6 \\
\hline Sem resposta & 1 & 0,2 \\
\hline Total & 533 & 100,0 \\
\hline \multicolumn{3}{|l|}{ Prazer em perigo nas relações sexuais } \\
\hline Sim & 139 & 26,1 \\
\hline Não & 389 & 73,0 \\
\hline Sem resposta & 5 & 0,9 \\
\hline Total & 533 & 100,0 \\
\hline \multicolumn{3}{|l|}{ Gosta de sexo em público } \\
\hline Sim & 181 & 34,7 \\
\hline Não & 345 & 64,7 \\
\hline Sem resposta & 7 & 1,3 \\
\hline \multicolumn{3}{|l|}{ Frequenta cinema pornô } \\
\hline Raramente & 118 & 22,1 \\
\hline Nunca-SR & 368 & 69,0 \\
\hline Total & 533 & 100,0 \\
\hline \multicolumn{3}{|l|}{ Frequenta banheiros públicos } \\
\hline Sempre/Frequentemente & 65 & 12,2 \\
\hline Raramente & 123 & 23,1 \\
\hline Nunca-SR & 345 & 64,7 \\
\hline Total & 533 & 100,0 \\
\hline \multicolumn{3}{|l|}{ Frequenta bares } \\
\hline Sempre/Frequentemente & 232 & 43,5 \\
\hline Raramente & 194 & 36,4 \\
\hline Nunca-SR & 107 & 20,1 \\
\hline Total & 533 & 100,0 \\
\hline \multicolumn{3}{|l|}{ Frequenta boates } \\
\hline Sempre/Frequentemente & 241 & 45,2 \\
\hline Raramente & 176 & 33,0 \\
\hline Nunca-SR & 116 & 21,8 \\
\hline Total & 533 & 100,0 \\
\hline
\end{tabular}


Tabela 1. Continuação

Table 1. Continuation

\begin{tabular}{|c|c|c|}
\hline Variável & $\mathrm{n}$ & $\%$ \\
\hline \multicolumn{3}{|l|}{ Frequenta praias } \\
\hline Sempre/Frequentemente & 123 & 23,1 \\
\hline Raramente & 205 & 38,5 \\
\hline Nunca-SR & 205 & 38,5 \\
\hline Total & 533 & 100,0 \\
\hline \multicolumn{3}{|l|}{ Frequenta clubes } \\
\hline Sempre/Frequentemente & 12 & 2,3 \\
\hline Raramente & 46 & 8,6 \\
\hline Nunca-SR & 475 & 89,1 \\
\hline Total & 533 & 100,0 \\
\hline \multicolumn{3}{|c|}{ Sente-se em risco de contrair HIV/Aids } \\
\hline Médio & 135 & 25,3 \\
\hline Alto & 66 & 12,4 \\
\hline Sem resposta & 12 & 2,3 \\
\hline Total & 533 & 100,0 \\
\hline \multicolumn{3}{|l|}{ Frequenta Saunas } \\
\hline Sempre/Frequentemente & 66 & 12,4 \\
\hline Raramente & 1544 & 28,9 \\
\hline Nunca-SR & 313 & 58,7 \\
\hline Total & 533 & 100,00 \\
\hline \multicolumn{3}{|l|}{ Frequenta Quarto escuro } \\
\hline Sempre/Frequentemente & 50 & 9,4 \\
\hline Raramente & 163 & 30,6 \\
\hline Nunca-SR & 320 & 60,0 \\
\hline Total & 533 & 100,0 \\
\hline \multicolumn{3}{|l|}{ Frequenta Videolocadoras } \\
\hline Sempre/Frequentemente & 77 & 14,4 \\
\hline Raramente & 192 & 36,0 \\
\hline Nunca-SR & 264 & 49,5 \\
\hline Total & 533 & 100,0 \\
\hline \multicolumn{3}{|l|}{ Classe social } \\
\hline Alta & 466 & 87,4 \\
\hline Média & 46 & 8,6 \\
\hline Baixa & 21 & 3,9 \\
\hline Total & 533 & 100,0 \\
\hline \multicolumn{3}{|l|}{ Raça/cor } \\
\hline Negra & 121 & 22,7 \\
\hline Outras & 28 & 5,3 \\
\hline Sem resposta & 3 & 0,6 \\
\hline Total & 533 & 100,0 \\
\hline \multicolumn{3}{|l|}{ Escolaridade } \\
\hline Universitário incompleto & 168 & 31,5 \\
\hline Universitário completo & 145 & 27,2 \\
\hline Pós-graduação & 104 & 19,5 \\
\hline Total & 533 & 100,0 \\
\hline \multicolumn{3}{|l|}{ Situação conjugal } \\
\hline Casado com mulher & 26 & 4,9 \\
\hline Solteiro/Separado-Divorciado & 366 & 68,7 \\
\hline Sem resposta & 9 & 1,7 \\
\hline Total & 533 & 100,00 \\
\hline
\end{tabular}


Tabela 2. Associação entre as variáveis do estudo e sexo anal desprotegido

Table 2. Association between the variables of the study and unprotected anal sex

\begin{tabular}{|c|c|c|c|}
\hline \multirow[b]{2}{*}{ Fator } & \multicolumn{2}{|l|}{ Sexo anal desprotegido } & \multirow[b]{2}{*}{$\mathrm{p}$} \\
\hline & Parceiro HIV +/ Sorologia desconhecida & HIV negativo & \\
\hline \multicolumn{4}{|l|}{ Idade } \\
\hline Até 30 anos & 81 & 207 & 0,152 \\
\hline Mais de 30 anos & 83 & 162 & \\
\hline Total & 164 & 369 & \\
\hline \multicolumn{4}{|l|}{ Raça/cor } \\
\hline Branca & 112 & 269 & 0,279 \\
\hline Não branca & 51 & 98 & \\
\hline Total & 163 & 367 & \\
\hline \multicolumn{4}{|c|}{ Número de parceiros homens no Carnaval } \\
\hline Até 1 & 110 & 275 & 0,026 \\
\hline Mais de 1 & 43 & 65 & \\
\hline Total & 153 & 340 & \\
\hline \multicolumn{4}{|c|}{ Número de parceiros homens no ano } \\
\hline Até 3 & 61 & 217 & 0,000 \\
\hline Mais de 3 & 95 & 145 & \\
\hline Total & 156 & 362 & \\
\hline \multicolumn{4}{|l|}{ Você já fez o teste do HIV? } \\
\hline Sim & 153 & 317 & 0,018 \\
\hline Não & 11 & 51 & \\
\hline Total & 164 & 368 & \\
\hline \multicolumn{4}{|c|}{ Sente-se em risco de contrair HIV/AIDS } \\
\hline Pouco & 73 & 247 & 0,000 \\
\hline Médio/Alto & 89 & 112 & \\
\hline Total & 162 & 359 & \\
\hline \multicolumn{4}{|l|}{ Sexo oral passivo } \\
\hline Gosto/Gosto muito & 146 & 349 & 0,038 \\
\hline Não gosto & 6 & 10 & \\
\hline Não sei-SR & 12 & 10 & \\
\hline Total & 164 & 369 & \\
\hline Frequenta videolocadoras & & & 0,184 \\
\hline Sempre/Frequentemente & 24 & 53 & \\
\hline Raramente & 50 & 142 & \\
\hline Nunca-SR & 90 & 174 & \\
\hline Total & 164 & 369 & \\
\hline \multicolumn{4}{|l|}{ Frequenta saunas } \\
\hline Sempre/Frequentemente & 27 & 39 & 0,007 \\
\hline Raramente & 57 & 97 & \\
\hline Nunca-SR & 80 & 233 & \\
\hline Total & 137 & 350 & \\
\hline \multicolumn{4}{|l|}{ Frequenta praias } \\
\hline Sempre/Frequentemente & 30 & 93 & 0,198 \\
\hline Raramente & 69 & 136 & \\
\hline Nunca-SR & 65 & 140 & \\
\hline Total & 164 & 369 & \\
\hline \multicolumn{4}{|l|}{ Frequenta banheiros públicos } \\
\hline Sempre/Frequentemente & 25 & 40 & 0,001 \\
\hline Raramente & 52 & 71 & \\
\hline Nunca-SR & 87 & 257 & \\
\hline Total & 164 & 368 & \\
\hline \multicolumn{4}{|l|}{ Frequenta cinema pornô } \\
\hline Sempre/Frequentemente & 16 & 31 & 0,019 \\
\hline Raramente & 48 & 70 & \\
\hline Nunca-SR & 100 & 268 & \\
\hline Total & 164 & 369 & \\
\hline
\end{tabular}


Tabela 3. Análise multivariada dos fatores associados com a prática do sexo anal com homens HIV positivo ou com homens de sorologia desconhecida

Table 3. Multivariate analysis of the factors associated with the practice of the anal sex with men positive HIV or with men of ignored HIV status

\begin{tabular}{|c|c|c|c|c|c|}
\hline Fator & Categorias & $\begin{array}{c}\text { OR } \\
\text { Bruto }\end{array}$ & $\begin{array}{c}\text { OR } \\
\text { IC } 95 \% \\
\end{array}$ & $\begin{array}{c}\text { OR } \\
\text { Ajustado }\end{array}$ & $\begin{array}{c}\text { OR } \\
\text { IC } 95 \% \\
\end{array}$ \\
\hline \multicolumn{6}{|c|}{ Numero de parceiros homens no Carnaval } \\
\hline & Ate 1 & 1 & & & \\
\hline & Mais de 1 & 1,65 & $(1,06-2,58)^{\ddagger}$ & 1,23 & $(0,74-2,04)$ \\
\hline \multicolumn{6}{|c|}{ Numero de parceiros homem no ano } \\
\hline & Até 3 & 1 & & & \\
\hline & Mais de 3 & 2,33 & $(1,58-3,42)^{\ddagger}$ & 1,71 & $(1,10-2,68)^{\ddagger}$ \\
\hline \multicolumn{6}{|c|}{ Você já fez o teste do HIV? } \\
\hline & Não & 1 & & & \\
\hline & Sim & 2,24 & $(1,83-3,94)$ & 0,60 & $(0,29-1,25)$ \\
\hline \multicolumn{6}{|c|}{ Sente-se em risco de contrair HIV/AIDS } \\
\hline & Pouco & 1 & & & \\
\hline & Médio/Alto & 2,69 & $(1,83-3,94)^{\ddagger}$ & 2,33 & $(1,53-3,57)^{\ddagger}$ \\
\hline \multicolumn{6}{|c|}{ Sexo oral passivo } \\
\hline & Não gosto & 1 & & & \\
\hline & Gosto/gosto muito & 0,46 & $(0,24-0,90)$ & 0,35 & $(0,16-0,76)^{\ddagger}$ \\
\hline \multicolumn{6}{|c|}{ Frequenta saunas } \\
\hline & Nunca-SR/ Raramente & 1 & & & \\
\hline & Sempre/Frequentemente & 1,67 & $(0,98-2,83)$ & 1,06 & $(0,54-2,07)$ \\
\hline \multicolumn{6}{|c|}{ Frequenta banheiros públicos } \\
\hline & Nunca-SR/ Raramente & 1 & & & \\
\hline & Sempre/Frequentemente & 1,47 & $(0,86-2,52)$ & 1,88 & $(0,98-3,59)$ \\
\hline \multicolumn{6}{|c|}{ Frequenta cinema pornô } \\
\hline & Nunca-SR/ Raramente & 1 & & & \\
\hline & Sempre/Frequentemente & 1,18 & $(062-2,22)$ & 0,60 & $(0,27-1,36)$ \\
\hline
\end{tabular}

HSH e também se apresentam coerentes com o perfil dos usuários da internet no Brasil ${ }^{11-13}$.

O número de parceiros sexuais durante os últimos doze meses aparece como um importante fator para o envolvimento em prática do sexo anal sem proteção ${ }^{1,2,7,18-22-}$ $, 24,27,29,30$. Porém, uma dificuldade na comparação dos resultados para este fator é o período escolhido para investigar a quantidade de parceiros, pois os estudos apresentam uma variação muito grande (2 até 12 meses). O número médio foi de 23 parceiros nos últimos 12 meses e está muito acima do que a literatura apresenta ${ }^{2,7,17,19,22}$. E entre os que relataram sexo anal desprotegido com homens HIV positivos ou de sorologia desconhecida, a média foi de 26 parceiros; e os que praticaram sexo anal sem preservativo com homens de sorologia negativa tiveram em média 20 parceiros.

Quantoà autopercepção derisco, poucos estudos com homens usuários da internet exploraram estefator em suas análises ${ }^{5,18,24,30}$, o mais comum é associar esta percepção ao uso de drogas e outros fatores intermediários que podem influenciar no envolvimento em práticas de risco para a infecção por HIV. Mas destacamos que neste trabalho a autopercepção aparece como um fator importante na presença dos demais fatores 
estudados, mas chama atenção o fato de a percepção alta e média do risco elevar as chances $(\mathrm{OR}=2,7)$ de envolvimento na prática do sexo anal desprotegido.

Gostar de sexo oral passivo associou-se ao envolvimento da prática do sexo anal sem proteção com parceiros HIV positivos ou de sorologia desconhecida, sendo que gostar de sexo oral passivo aparece como fator de proteção para a prática do sexo anal desprotegido. Na literatura internacional, estudos que coletaram dados na internet e em locais de sociabilidade gay também encontraram associações similares ${ }^{1,2,5,7,17-}$ 26,29,30. Alguns desses estudos compararam grupos de HSH soropositivo e negativo para HIV, analisando o sexo anal insertivo sem o uso do preservativo e sexo com parceiro sorodiscordante, e nestes estudos, o grupo que recruta parceiros sexuais na internet apresentou maior risco de envolvimento em práticas sexuais desprotegidas ${ }^{5-7,23,26}$.

Frequentar locais específicos de sociabilidade gay, como saunas, banheiros públicos e cinemas pornôs, com o objetivo de encontrar parceiros sexuais e a associação com a prática do sexo anal sem proteção é demonstrada em estudos internacionais que recrutaram HSH na internet $t^{2,3,7,17-20,27}$ ou em outros locais da "cena" gay ${ }^{2,27}$. Porém, na nossa análise multivariada, esse fator não atingiu níveis de significância estatística.

$\mathrm{O}$ ambiente da internet vem sendo considerado um facilitador para o encontro de parceiros sexuais, em especial para os $\mathrm{HSH}$ que buscam o anonimato, a não exposição, e procuram envolvimento em práticas sexuais mais arriscadas e/ou sexo anal desprotegido $0^{4,6,19-22}$. Por exemplo, a literatura aponta que o número de parceiros sexuais nos últimos 12 meses é maior entre os $\mathrm{HSH}$ que procuram parceiros sexuais na internet, quando comparados com homens que buscam parceria fora da rede ${ }^{22}$. Horvath et al. encontraram diferença estatisticamente significante entre HSH que buscavam parceiros sexuais ${ }^{22}$ on-line $(31,1 \%)$, offline $(30,7 \%)$ e on-line/offline (48,9\%).

Em uma revisão de estudos realizados com $\mathrm{HSH}^{6}$, os autores mostraram que os participantes recrutados on-line têm mais chance de buscar parceria sexual na internet (OR=9,37; IC 95\% 5,30-16,57). Para a prática do sexo anal desprotegido, encontraram que a chance de envolvimento para os homens usuários da internet foi uma vez e meia maior (OR=1,57; IC 95\% 1,25-1,98) quando comparados com os HSH que buscam parceiros em bares e em outros locais. Também encontraram uma porcentagem maior de envolvimento na prática do sexo anal desprotegido entre 78,3 e $93,5 \%$ para os homens que usam a internet para buscar sexo, nos estudos analisados. Entre os homens HIV negativos a busca por sexo na internet foi entre 81,9 e $90,5 \%$, e o envolvimento na prática do sexo anal desprotegido foi de 22,1 a 26,9\%. Nestes estudos observou-se que $28,8 \%$ dos participantes relataram algum tipo de DST.

Não foi possível verificar a relação entre outros fatores considerados importantes no contexto das práticas sexuais desprotegidas entre $\mathrm{HSH}$, como o tipo de parceiro (ocasional ou fixo), uso de álcool e drogas ilícitas, bem como aspectos da violência e homofobia, pois não estavam disponíveis no questionário. Os resultados deste trabalho não podem ser generalizados para outros HSH, por se tratar de uma amostra de conveniência composta por voluntários usuários da internet. Porém, estas informações são importantes, pois refletem algumas das práticas e comportamentos sexuais, bem como características de uma parte dos HSH que usam a internet para procurar parceiros sexuais. Além disso, no Brasil, poucos estudos têm se preocupado em recrutar e investigar os comportamentos sexuais de homens com práticas homoeróticas na internet, metodologia de recrutamento amplamente utilizada em estudos em outros países desde 2001.

\section{Agradecimentos}

Somos gratos à equipe do Projeto Convida (Luís Augusto Vasconcelos da Silva, José Carlos Oliveira Chaves, Ivone Meira, Rosangela Castro e Iara Martinez), pelo 
trabalho de campo. À Agência Nacional de Pesquisa em Aids (ANRS) da França, pelo financiamento, e ao /MS pela viabilização da pesquisa na Cooperação Brasil-França. A Sandra Reis, estudante de estatística da
UFBA, pela organização dos dados. Ao Centro de Estudos de AIDS do Rio Grande do Sul - CEARGS. E aos homens que voluntariamente responderam ao questionário da pesquisa na internet.

\section{Referências}

1. Coleman E, Horvath K, Miner M, Ross MW, Oakes M, Rosser S. Compulsive Sexual Behavior and Risk for Unsafe Sex Among Internet Using Men Who Have Sex with Men. Arch Sex Behavior. 2009; July; DOI:10.1007/s10508-0099507-5. Available at: < http://www.springerlink.com/ content/6818twp172p6m31m . Accessed: november, 2009.

2. Reisner SL, Mimiaga JM, Skeer M, VanDerwarker R, Gaucher MJ, O'Connor CA, et al. Differential HIV Risk Behavior among Men who have Sex with Men Seeking Health-Related Mobile Van Services at Diverse Gay-Specific Venues. AIDS Behavior. 2009;13:822-831.

3. Bolding G, Davis M, Hart G, Sherr L, Elford J. Where Young MSM Meet Their First Sexual Partner: the Role of the Internet. AIDS Behavior. 2007;11:522-526.

4. Centers for diseas and Control e Prevention (US). HIV/ AIDS Fact Sheet: HIV/AIDS among Men Who Have Sex with Men. 2007. Available at: <http://www.cdc.gov/hiv/ topics $/ \mathrm{msm} /$ resources/factsheets $/ \mathrm{msm} . \mathrm{htm}>$. Accessed: june 22, 2007.

5. Kesteren NMC, HOSPERS HJ, KOK G. Sexual risk behavior among HIV-positive men who have sex with men: a literature review. Patient Education and Couseling. 2007;65:5-20.

6. Liau A, Millett G, Marks G. Meta-analytic Examination of Online Sex-Seeking and Sexual Risk Behavior Among Men Who Have Sex With Men. Sexually Transmitted. Diseases. 2006;33(9):576-584.

7. Chiasson, MA, Hirshfield S, Remien RH, Humberstone M, Wong T, Wolitski RJ. A Comparison of On-Line and Off-Line Sexual Risk in Men Who Have Sex With Men An Event-Based On-Line Survey. Journal Acquired Immune Deficiency Syndromes. 2007;44(2):235-243.

8. Strazza L, Azevedo RS, Carvalho HB. Comportamento de risco para uso de drogas e infecção pelo HIV: questionário na internet acoplado com textos educacionais para língua portuguesa. Revista da Sociedade Brasileira de Medicina Tropical. 2007;4(40):400-404.

9. da Silva LAV. Desejo à Flor da Tel@: a relação entre risco e prazer nas práticas de Barebacking [tese de doutorado]. Salvador: Instituto de Saúde Coletiva/Universidade Federal da Bahia, 2008.

10. IBGE, Instituto Brasileiro de Geografia e Estatística. Ministério do Planejamento, Orçamento e Gestão. Pesquisa
Nacional de Amostragem por domicílio (PNAD), Síntese de Indicadores Sociais, Rio de Janeiro; 2005.

11. IBGE, Instituto Brasileiro de Geografia e Estatística. Ministério do Planejamento, Orçamento e Gestão. Pesquisa Nacional de Amostragem por domicílio (PNAD), Síntese de Indicadores Sociais, Rio de Janeiro; 2006.

12. IBGE, Instituto Brasileiro de Geografia e Estatística. Ministério do Planejamento, Orçamento e Gestão. Pesquisa Nacional de Amostragem por domicílio (PNAD), Síntese de Indicadores Sociais, Rio de Janeiro; 2008.

13. Dourado I, Vasconcelos LA, Iriart J, Stangl A, Chaves JC, Adam P, et al. Projeto CONVIDA - Estudo sobre conhecimentos, atitudes, comportamentos e práticas de risco para a infecção pelo HIV entre homens que fazem sexo com homens. Universidade Federal da Bahia Instituto de Saúde Coletiva. Salvador; 2003.

14. Mann JM, Tarantola DJM, editors. AIDS in the World II.Global Dimensions, Social Roots and Responses. NewYork/Oxford: Oxford University Press; 1996. p 441-3.

15. Ayres, JRCM. et al. O conceito de vulnerabilidade e as práticas de saúde: novas perspectivas e desafios. In: Czeresnia DFCM (Org.). Promoção da saúde: conceitos, reflexões, tendências. Rio de Janeiro: Editora Fiocruz; 2003. p. 117-139.

16. Barros AJD, Victora CG. A nationwide wealth score based on the 2000 Brazilian demographic census. Revista de Saúde Pública. 2005;4(39):523-529.

17. Folch C, Muñoz R, Zaragoza K et al. Sexual risk behaviour and its determinants among men who have sex with men in Catalonia, Spain. Euro Surveillance. 2009;14(47). Available at:<http://www.eurosurveillance.org/ViewArticle. aspx?ArticleId=19415> Accessed January 10, 2010.

18. Zablotska IB, Crawford J, Imrie J, Prestage G, Jin F, Grulich A, et al. Increases in Unprotected Anal Intercourse with Serodiscordant Casual Partners Among HIV-Negative Gay Men in Sydney. AIDS Behavior. 2009;13:638-644.

19. Rosser BRS, Miner MH, Bockting WO, Ross MW, Konstan J, Gural L, et al. HIV Risk and the Internet: Results of the Men's Internet Sex (MINTS) Study. AIDS Behavior. 2009;13:746-756.

20. Ogilvie G, Taylor D, Trussler T, Marchand R, Gilbert M, Moniruzzaman A, et al. Seeking sexual partners on the 
Internet: a marker for risk sexual behavior in men who have sex with men. Can J. Public Health. 2008;99(3):185-188.

21. Akin M, Fernández MI, Bowen GS, Warren JC. HIV risk behavior of Latin American and Caribbean men who have sex with men in Miani, Florida, USA. Rev. Panam Salud Public. 2008;23(5):341-348.

22. Horvath KJ, Rosser BRS, Remafedi G. Sexual Risk Taking Among Young Internet-Using Men Who Have Sex With Men. American Journal of Public Health. 2008;6(98):1059-1067.

23. Horvath KJ, Nygaard K, Rosser BRS. Ascertaining Partner HIV Status and its Association with Sexual Risk Behavior Among Internet-Using Men Who Have Sex with Men. AIDS Behavior. 2009; nov 17. DOI 10.1007/s10461-009-9633-z.

24. Mackellar D, Valleroy L, Behel S, Secura G, Bingham T, Celentano D, et al. Unintentional HIV exposures from young men who have sex with men who disclose being HIV negative. Aids. 2006;20(12):1637-1644.

25. Chiasson MA, Parsons JT, Tesoriero JM et al. HIV Behavioral Research Online. Journal of Urban Health: Bulletin of the New York Academy of Medicine. 2006;83(1):73-85.

26. Elfort J, Bolding G, Davis M, Sherr L, Hart G. Web-Based Behavioral Surveillance Among Men Who Have Sex With Men: A Comparison of Online and Offline Samples in London, UK. J Acquir Immune Defic Syndr. 2004;35(4):421-426.

27. Raymond HF, Rebchook G, Curotto A, Vaudrey J, Amsden M, Levine D, et al. Comparing Internet-Based and Venue-Based Methods to Sample MSM in the San Francisco Bay Area. Aids Behavior. 2009. DOI 10.1007/s10461-009-9521-6.

28. Crosby R, Diclemente RJ, Mettey, A. Correlates of Recent Unprotected Anal Sex among Men Having Sex With
Men Attending a Large Sex Resort in the South. Sexually Transmitted Diseases. 2003;30(12):909-913.

29. Klein H. HIV risk practices sought by men who have sex with other men, and who use internet websites to identify potential sexual partners. Sex Health. 2008;5(3):243-250.

30. Mimiaga MJ, Goldhammer H, Belanoff C, Ashley MT, Kenneth HM. Men Who Have Sex With Men: Perceptions About Sexual Risk, HIV and Sexually Transmitted Disease Testing, and Provider Communication. Sexually Transmitted Diseases. 2007;34(2):113-119.

31. Horvath KJ, Beadnell B, Bowen AM. A Daily Web Diary of the Sexual Experiences of Men who have Sex with Men: Comparisons with a Retrospective Recall Survey. AIDS Behavior. 2007;11:537-548.

32. Bolding G, Davis M, Hart G, Sherr L, Elford J. Gay men who look for sex on the Internet: is there more HIV/STI risk with online partners? AIDS. 2005;19:961-968.

33. Brito AM, Castilho EA, Szwarcwald CL. AIDS e infecção pelo HIV no Brasil: uma epidemia multifacetada. Revista da Sociedade Brasileira de Medicina Tropical. 2001;34(2):207-217.

34. Vieira de Souza et al. The association of socioeconomic status and use of crack/cocaine with unprotected anal sex in a cohort of men who have sex with men in Rio de Janeiro, Brazil. In: VIEIRA de SOUZA. Características sóciodemográficas, comportamentais e vulnerabilidade à infecção pelo vírus da imunodeficiência humana em homens que fazem sexo com homens do "projeto Rio". [tese de doutorado]. Rio de Janeiro: Fundação Oswaldo Cruz, Escola Nacional de Saúde Pública; 2001.

Recebido em: 19/04/2010 Versão final apresentada em: 07/01/2011 Aprovado em: 28/02/2011 